\title{
PARASITEMIA CONSTANTE DURANTE 24 HORAS CONSECUTIVAS NA INFECÇÃO EXPERIMENTAL PELO TRYPANOSOMA CRUZI*
}

\begin{abstract}
Ítalo A. Sherlock
Observou-se a parasitemia horária do Trypanosoma cruzi através de hemoscopiase de xenodiagnósticos horários, em camundongos na fase aguda da infecção e em cobaias e camundongos na fase crônica. O número de T. cruzi no sangue e o número de triatomineos positivos por xenodiagnósticos foi freqüente e variável mas não foi ciclico durante as 24 horas do dia. A qualquer hora, tanto na fase aguda como na crônica, o T. cruzi pôde ser detectado através hemoscopias exenos. Não foi constatado um ritmo circadiano para o T. cruzi.
\end{abstract}

Palavras chaves: Trypanosoma cruzi. Parasitemia horária. Ritmo circadiano. Xenodiagnóstico.

O ritmo circadiano e as modificações ciclicas nos niveis de parasitemias, expressam uma verdadeira seleção natural para assegurar o contato máximo entre o parasita e o vetor.

Talvez, como foi demonstrado para algumas espécies de tripanossomas, inclusive para o Trypanosoma minasense 45 , pudesse haver um ritmo nictemeral do Trypanosoma cruzi que, através da hemoscopia ou do xenodiagnóstico, facilitasse a detectação do flagelado em certos periodos do dia. Por outro lado, talvez permitisse correlacionar, através de suas atividades nos ecossistemas naturais, certas espécies de mamiferos que sảo encontradas naturalmente infectadas pelo $T$. cruzi com seus vetores e dai esclarecer seus papéis na epidemiologia da doença de Chagas.

As poucas observações realizadas sobre a parasitemia horária e sobre a positividade horária do xenodiagnóstico para o T. cruzi durante as 24 horas do dia, trazem poucos esclarecimentos para o fato 1121416 . Por esse motivo, realizamos experiências utilizando camundongos e cobaias em diferentes fases da infecção, durante as $\mathbf{2 4}$ horas do dia, na tentativa de demonstrar uma ritmicidade dos indices de parasitemia para o $T$. cruzi.

\section{MATERIAL E MÉTODOS}

Animais inoculados

Foram realizados dois tipos de experiências. Um primeiro tipo com 7 camundongos brancos com

\footnotetext{
* Centro de Pesquisas Gonçalo Moniz - FIOCRUZ - Rua Valdemar Falcão 121, 40000 Salvador, Bahia.
}

Recebido para publicação em 27/2/84. pesos aproximados, identificados por ordem alfabética de $A$ a $G$, em fase aguda da infecção, cerca de 10 dias após a inoculação, quando a parasitemia era elevada. Hemoscopias e xenodiagnósticos horários simultâneos com Rhodnius neglectus foram feitos somente nos camundongos ABC. Os camundongos D E F G submeteram-se apenas a hemoscopias horárias. Esse primeiro experimento durou 72 horas consecutivas de três dias, utilizando-se os camundongos com periodos de repouso intercalados, conforme o esquema que adiante apresentamos.

Um segundo tipo de experiência, foi feito com 3 cobaias e 10 camundongos, quando tinham 90 dias de inoculados e a hemoscopia mostrava escassos tripanossomas circulantes. Este experimento foi realizado com todos os animais durante 24 horas consecutivas e neles foram feitos simultaneamente hemoscopias e xenodiagnósticos com ninfas em 10 estágio de Panstrongylys megistus. Os animais inoculados tinham aproximadamente o mesmo peso corporal, respectivamente nos grupos de roedores e cobaias.

\section{Cepa de Trypanosoma cruzi}

A cepa de tripanossoma usada nos dois tipos de experiência e que tinham as caracteristicas morfológicas e biológicas do $T$. cruzi, foi isolada de uma cobaia encontrada naturalmente infectada, numa residência da área de São Felipe, Bahia. Cerca de 70.000 tripanossomas contados pelo método de Brener ${ }^{5}$, foram inoculados por via intraperitoneal nos animais sadios. A confirmação das infecções foi feita através do encontro do flagelado no sangue colhido da cauda dos camundongos e da orelha das cobaias. 
Sherlock IA. Parasitemia constante durante 24 horas consecutivas na infecção experimental pelo Trypanosoma cruzi. Revista da Sóciedade Brasileira de Medicina Tropical 17: 137-144, Jul-Set, 1984

\section{Triatomineos}

Os triatomineos utilizados foram provenientes das colònias que mantinhamos no laboratório. No primeiro experimento, para os camundongos ABC usamos um total de 250 ninfas de $R$. neglectus em 4 . ou 5 . estágios, 5 por camundongo, em cada horário.

Como uma ninfa de $R$. neglectus $40^{\circ}$ ou 5: estágio suga de cada vez de 90 a $120 \mathrm{mg}$ de sangue ${ }^{2}$, para evitar a espoliação sangüínea dos roedores, no segundo experimento, passamos a utilizar de ninfas de 1. estágio de $P$. megistus, que tinhamos observado, naquela oportunidade, se infectarem excelentemente bem, tanto em camundongos como em cobaias. Estas ninfas, desde recém-nascidas, eram mantidas em jejum por 10 dias, sendo usado o total de 1.720 exemplares, cerca de 15 ninfas por animal de cada horário, para essa experiência com os 10 camundongos e as 3 cobaias.

\section{Xenodiagnósticos}

Os xenos eram feitos de hora em hora, por duas pessoas, revezadas por outras duas, a cada turno de 6 horas de trabalho continuo. Logo após colhido o sangue para a hemoscopia, colocavam-se os triatomineos para sugar diretamente sobre o animal do horário (cobaia ou camundongos), estando o animal contido num cilindro de tela de arame de malhas largas. Este era então colocado dentro de um vasilhame de vidro onde estavam os triatomíneos. Após 30 minutos, os triatomineos engurgitados que se haviam alimentado, eram retirados e guardados em tubos de vidro com suportes de papel de filtro e com as devidas anotaçōes. Após 20 dias, os tubos digestivos dos triatomineos eram examinados ao microscópio, após a dissecção em solução salina, entre lâmina e lamínula.

\section{Hemoscopias}

Para as hemoscopias horárias o sangue era colhido, da cauda do camundongo ou da orelha da cobaia, após um pequeno corte com tesoura, por meio de microtubos heparinizados para microhematócritos. Estes tubos da marca "Pre-Cal-Adams", eram calibrados para $77 \mathrm{~mm} \pm 0,5$ de altura e $0,55 \pm 0,05$ de diâmetro interno, enchendo-se de sangue até a marca $15 \mathrm{~mm}$ de altura, fornecendo, portanto, um volume de $3,09 \mathrm{~mm}^{3}$, de acordo com a fórmula $V=\Pi R^{2} h$. Logo após colhido, o sangue era espalhado numa lâmina com uma gota de liquido de Errecart e após seco, era fixado e corado pelo Giemsa. Os tripanossomas eram cuidadosamente contados ao microscópio, em todo o conteúdo do esfregaço.

Das .cobaias era possivel colherem-se duas porções de sangue: uma parte servia para o exame do esfregaço corado pelo Giemsa e a outra para o exame imediato a fresco em solução salina. Neste segundo método, cometemos freqüentes erros de contagem, devido principalmente ao movimento do parasita e a pressa que o experimento exigia. Por este motivo, os resultados deste último tipo de exame não foram levados em consideração para a análise final quantitativa. $\mathrm{O}$ seu valor foi mais de confirmação qualitativa da positividade horária.

\section{Horários de Investigação}

No primeiro experimento, devido ao receio de exaurir a volemia de todos os camundongos e ter a experiência anulada, as hemoscopias com xenos simultâneos foram feitos somente nos 3 camundongos $A B$ e $C$, enquanto que nos outros 4 camundongos D E F $G$ foram feitas somente hemoscopias nos horários consecutivo, conforme já mencionamos.

Para os camundongos ABC tivemos de lançar mão de um esquema de utilização dos animais por periodos de 6 horas, intercalados por periodos de 12 horas de repouso, durante o tempo total das 72 horas de observação de cada animal. Desta forma foi possivel observar a parasitemia de cada camundongo do grupo $\mathrm{ABC}$, nos quatro turnos de 6 horas que compõem as 24 horas de um dia, conforme o esquema a seguir:

Horas e Camundongos

\begin{tabular}{lcccc}
\hline & $\begin{array}{c}13 h \text { às } \\
19 h\end{array}$ & $\begin{array}{c}19 h a ̀ \\
01 h\end{array}$ & $\begin{array}{c}01 h a ̀ s \\
07 h\end{array}$ & $\begin{array}{c}07 h a ̀ s \\
13 h\end{array}$ \\
\hline $10 \mathrm{dia}$ & $\mathrm{A}$ & $\mathrm{B}$ & $\mathrm{C}$ & $\mathrm{A}$ \\
$20 \mathrm{dia}$ & $\mathrm{B}$ & $\mathrm{C}$ & $\mathrm{A}$ & $\mathrm{B}$ \\
$30 \mathrm{dia}$ & $\mathrm{C}$ & $\mathrm{A}$ & $\mathrm{B}$ & $\mathrm{C}$ \\
\hline
\end{tabular}

No segundo experimento, tanto as cobaias como os camundongos foram testados com hemoscopias e xenos simultaneos durante as 24 horas consecutivas de um dia, tendo os animais apenas. 20 minutos de repouso em cada horário, que correspondia ao término do horário de um xeno e começo do xeno do horário seguinte. Nos intervalos, os animais eram 
Sherlock IA. Parasitemia constante durante 24 horas consecutivas na infecção experimental pelo Trypanosoma cruzi. Revista da Sociedade Brasileira de Medicina Tropical 17: 137-144, Jul-Set, 1984

colocados em caixas com alimento e água. Ambos os experimentos foram iniciados às 13 horas.

\section{RESULTADOS}

Nas Tabelas 1 e 2 estão os resultados obtidos na primeira experiência com xenos e hemoscopias na fase aguda da infecção. Houve positividade das hemoscopias em todos os horários do dia, com todos os animais observados. Fato semelhante ocorreu em quase todos os horários dos xenos feitos nos camundongos $\mathrm{ABC}$. As quedas nos indices de positividade dos xenos nestes camundongos, foram irregulares e não coincidiram nos três animais observados. $\mathrm{Da}$ mesma forma, não foi verificada a existência de maiores indices de positividade dos xenos para determinados periodos do dia.

As hemoscopias, embora positivas em qualquer hora do dia ou da noite, mostraram irregularidade para o número de tripanossomas, não havendo contudo predominância do número de flagelados em determinados horários.

No segundo experimento, quando a infecção era mais crônica e o número de tripanossomas no sangue era muito baixo, os resultados parecem ter o mesmo significado da experiência anterior. Tanto nas cobaias como nos camundongos, conforme os dados da Tabela 5 , as hemoscopias também demonstraram números

Tabela 1-Positividade de xenodiagnóstico com Rhodnius neglectus em camundongos na fase aguda da infecção pelo Trypanosoma cruzi.

\begin{tabular}{|c|c|c|c|c|c|c|c|c|c|}
\hline \multirow{3}{*}{$\begin{array}{c}\text { Hora } \\
\text { do } \\
\text { dia }\end{array}$} & \multicolumn{2}{|c|}{ Camundongo $A(+)$} & \multicolumn{2}{|c|}{ Camundongo $B(+)$} & \multicolumn{2}{|c|}{ Camundongo $C(+)$} & \multicolumn{2}{|c|}{ Total } & \multirow[b]{3}{*}{$\%$} \\
\hline & \multicolumn{2}{|c|}{ No Ninfas } & \multicolumn{2}{|c|}{ No Ninfas } & \multicolumn{2}{|c|}{ No Ninfas } & \multicolumn{2}{|c|}{ No Ninfas } & \\
\hline & $E x$ & Pos. & $E x$. & Pos. & $E x$ & Pos. & $E x$ & Pos. & \\
\hline 14 & 3 & 3 & 3 & 1 & 3 & 1 & 9 & 5 & 55,5 \\
\hline 15 & 3 & 3 & 3 & 3 & 3 & 1 & 9 & 7 & 78 \\
\hline 16 & 3 & 3 & 3 & 3 & 3 & 3 & 9 & 9 & 100 \\
\hline 17 & 3 & 1 & 3 & 3 & 3 & 0 & 9 & 4 & 44,4 \\
\hline 18 & 3 & 3 & 2 & 2 & 3 & 1 & 8 & 6 & 75 \\
\hline 19 & 3 & 3 & 3 & 3 & 3 & 0 & 9 & 6 & 67 \\
\hline 20 & 3 & 2 & 3 & 2 & 3 & 3 & 9 & 7 & 78 \\
\hline 21 & 3 & 3 & 3 & 0 & 3 & 2 & 9 & 5 & 55 \\
\hline 22 & 3 & 1 & 3 & 3 & 3 & 2 & 9 & 6 & 67 \\
\hline 23 & 3 & 2 & 3 & 3 & 3 & 3 & 9 & 8 & 89 \\
\hline 24 & 3 & 2 & 3 & 3 & 3 & 2 & 9 & 7 & 78 \\
\hline 01 & 3 & 2 & 3 & 2 & $(++)$ & - & 6 & 4 & 67 \\
\hline 02 & 3 & 1 & 3 & 3 & 3 & 1 & 9 & 5 & 55,5 \\
\hline 03 & 3 & 2 & 3 & 3 & 3 & 2 & 9 & 7 & 78 \\
\hline 04 & 2 & 1 & 3 & 1 & 3 & 3 & 8 & 5 & 62,5 \\
\hline 05 & 3 & 2 & 5 & 4 & 3 & 2 & 11 & 8 & 73 \\
\hline 06 & 3 & 2 & 3 & 3 & 3 & 2 & 9 & 7 & 78 \\
\hline 07 & 5 & 3 & 3 & 1 & 3 & 3 & 11 & 7 & 64 \\
\hline 08 & 3 & 3 & 3 & 1 & 2 & 1 & 8 & 5 & 62,5 \\
\hline 09 & 3 & 3 & 3 & 1 & 3 & 1 & 9 & 5 & 55,5 \\
\hline 10 & 3 & 3 & 3 & 3 & 2 & 0 & 8 & 6 & 75 \\
\hline 11 & 3 & 3 & 3 & 3 & 3 & 1 & 9 & 7 & 78 \\
\hline 12 & 3 & 3 & 4 & 0 & 3 & 1 & 10 & 4 & 40 \\
\hline 13 & 3 & 1 & 1 & 1 & 3 & 0 & 7 & 2 & 28,5 \\
\hline Total & 73 & 55 & 72 & 52 & 67 & 35 & 212 & 142 & 67 \\
\hline
\end{tabular}

$(+)$ Correspondem aos camundongos ABC da Tabela 2.

$(++)$ Morreu durante a experiência. 
Sherlock IA. Parasitemia constante durante 24 horas consecutivas na infecção experimental pelo Trypanosoma cruzi Revista da Sociedade Brasileira de Medicina Tropical 17: 137-144, Jul-Set, 1984

Tabela 2 - Número de trigomastigotas por $3,09 \mathrm{~mm}^{3}$ de sangue nas horas do dia em camundongos infectados por Trypanosoma cruzi (fase aguda)

\begin{tabular}{|c|c|c|c|c|c|c|c|c|}
\hline \multirow{2}{*}{$\begin{array}{c}\text { Hora } \\
\text { do } \\
\text { dia }\end{array}$} & \multicolumn{8}{|c|}{ Camundongos e número de T. cruzi por $3,09 \mathrm{~mm}^{3}$ de sangue } \\
\hline & $A(+)$ & $B(+)$ & $C(+)$ & $D$ & $E$ & $F$ & $G$ & Total \\
\hline 14 & 244 & 294 & 275 & 873 & 741 & 88 & 17 & 2.532 \\
\hline 15 & 402 & 609 & 74 & 713 & 72 & 36 & 358 & 2.264 \\
\hline 16 & 277 & 1.010 & 759 & 470 & 173 & 99 & 129 & 2.917 \\
\hline 17 & 41 & 985 & 112 & 895 & 443 & 23 & 5 & 2.504 \\
\hline 18 & 421 & 821 & 19 & 1.151 & 22 & 13 & 46 & 2.493 \\
\hline 19 & 206 & 107 & 3 & 557 & 324 & 27 & $(* *)$ & 1.224 \\
\hline 20 & 25 & 51 & 196 & 120 & 17 & 32 & 81 & 522 \\
\hline 21 & 25 & 423 & 1.178 & 705 & 913 & 231 & 18 & 3.493 \\
\hline 22 & 98 & 64 & 2.121 & 949 & 1.408 & 22 & 42 & 4.704 \\
\hline 23 & 18 & 263 & 772 & 340 & 137 & 293 & 134 & 1.957 \\
\hline 24 & 49 & 622 & 1.926 & 240 & 186 & 22 & 76 & 3.121 \\
\hline 01 & 135 & 543 & 634 & 301 & 232 & 102 & 272 & 2.219 \\
\hline 02 & 435 & 15 & 249 & 30 & 186 & 194 & 341 & 1.450 \\
\hline 03 & 219 & 30 & 478 & 329 & 438 & 201 & 241 & 1.936 \\
\hline 04 & 1.147 & 19 & 454 & 611 & 268 & 344 & 351 & 3.194 \\
\hline 05 & 953 & $(* *)$ & 710 & 545 & 404 & 310 & 91 & 3.013 \\
\hline 06 & 322 & $(* *)$ & 288 & 158 & 231 & 317 & 188 & 1.504 \\
\hline 07 & 634 & 4 & 712 & 558 & 308 & 78 & 443 & 2.737 \\
\hline 08 & 260 & 245 & 19 & 1.092 & 696 & 59 & 52 & 2.423 \\
\hline 09 & 285 & 91 & 1 & 99 & 213 & 151 & 106 & 946 \\
\hline 10 & 204 & 200 & 32 & 937 & 595 & 54 & 154 & 2.176 \\
\hline 11 & 274 & 408 & 53 & 949 & 815 & 117 & 41 & 2.657 \\
\hline 12 & 375 & 762 & 254 & 1.094 & 453 & 72 & 24 & 3.034 \\
\hline 13 & 650 & 150 & 413 & 161 & 367 & 58 & 17 & 1.816 \\
\hline
\end{tabular}

(+) Correspondem aos camundongos ABC da Tabela 1.

(**) Material danificado.

indiferentes de tripanossomas em horários do dia porém, ao contrário da fase aguda, os números de parasitas eram baixissimos. Freqüentemente, não eram observados tripanossomas, principalmente nos camundongos. Da mesma forma, não houve horários especiais em que se demonstrasse maior número de tripanossomas. Os xenodiagnósticos também apresentaram resultados semelhantes aos da fase aguda, não havendo uma predominância de positividade para determinados horários, tanto para as cobaias como para os camundongos (Tabelas 3 e 4).

\section{DISCUSSÃO}

Apesar da importância prática do ritmo circadiano, fenômeno já demnnstrado para diversas especies de tripanossomas 7 \& 910111317 , o assunto quase não tem sido investigado para o $T$. cruzi, sendo a maioria dos trabalhos publicados a respeito de nossa autoria $^{1} 121416$. Provavelmente, tal fato liga-se às dificuldades que existem para a realização desse tipo de observação. Diversos fatores são envolvidos para o controle e padronização do experimento. Alguns desses fatores já são conhecidos, entre eles, a luz e a temperatura que interferem na periodicidade do Trypanosoma rotatorium 346111317 e também o sono, ainda não bem investigado, mas que possivelmente muito poderá influir na ritmicidade da parasitemia dos tripanossomas. Observações feitas com o Trypanosoma lewisi e Trypanosoma duttoni parecem sugerir haver influência do sono no ritmo circadiano dos flagelados 6 .

De acordo com Seed ${ }^{13}$, existe um ritmo estacional e diurno para o Trypanosoma rotatorium 
Sherlock IA. Parasitemia constante durante 24 horas consecutivas na infecção experimental pelo Trypanosoma cruzi. Revista da Sociedade Brasileira de Medicina Tropical 17: 137-144, Jul-Set, 1984

Tabela 3 - Positividade para Trypanosoma cruzi do xenodiagnóstico em cobaias infectadas durante 24 horas consecutivas, com ninfas de Panstrongylus megistus (fase crônica).

\begin{tabular}{crrrrrrrrr}
\hline Hora & \multicolumn{2}{c}{ Cobaia A } & \multicolumn{2}{c}{ Cobaia $B$} & \multicolumn{2}{c}{ Cobaia C } & \multicolumn{3}{c}{ Total } \\
\cline { 2 - 9 } do & \multicolumn{2}{c}{ N. Ninfas } & \multicolumn{2}{c}{ No Ninfas } & \multicolumn{2}{c}{ No Ninfas } & \multicolumn{2}{c}{ No Ninfas } & \\
dia & Ex. & Pos. & Ex. & Pos. & Ex. & Pos. & Ex. & Pos. & $\%$ \\
\hline 14 & 9 & 8 & 8 & 6 & 10 & 10 & 27 & 24 & 89 \\
15 & 7 & 3 & 2 & 2 & 2 & 2 & 11 & 7 & 64 \\
16 & 9 & 7 & 3 & 3 & 8 & 7 & 20 & 17 & 76 \\
17 & 7 & 6 & 6 & 6 & 5 & 4 & 18 & 16 & 89 \\
18 & 7 & 5 & 5 & 4 & 5 & 4 & 17 & 13 & 76 \\
19 & 6 & 5 & 10 & 7 & 5 & 4 & 21 & 16 & 76 \\
20 & 6 & 5 & 10 & 10 & 8 & 7 & 24 & 22 & 92 \\
21 & 8 & 8 & 4 & 2 & 7 & 5 & 19 & 15 & 79 \\
22 & 2 & 2 & 3 & 3 & 8 & 7 & 13 & 12 & 92 \\
23 & 4 & 4 & 6 & 6 & 2 & 2 & 12 & 12 & 100 \\
24 & 7 & 7 & 6 & 4 & 10 & 8 & 23 & 19 & 83 \\
01 & 7 & 6 & 6 & 6 & 4 & 4 & 17 & 16 & 94 \\
02 & 6 & 6 & 6 & 5 & 5 & 4 & 17 & 15 & 88 \\
03 & 4 & 3 & 7 & 6 & 7 & 7 & 18 & 16 & 89 \\
04 & 10 & 10 & 2 & 1 & 6 & 6 & 18 & 17 & 94 \\
05 & 7 & 7 & 4 & 4 & 7 & 7 & 18 & 18 & 100 \\
06 & 7 & 1 & 5 & 5 & 8 & 8 & 20 & 14 & 70 \\
07 & 3 & 2 & 7 & 6 & 9 & 9 & 19 & 17 & 89 \\
08 & 3 & 3 & 7 & 6 & 6 & 6 & 16 & 15 & 94 \\
09 & 7 & 5 & 4 & 3 & 3 & 3 & 14 & 11 & 78 \\
10 & 4 & 4 & 3 & 3 & 4 & 4 & 11 & 11 & 100 \\
11 & 8 & 8 & 6 & 6 & 4 & 4 & 18 & 18 & 100 \\
12 & 4 & 4 & 3 & 3 & 4 & 4 & 11 & 11 & 100 \\
13 & 3 & 3 & 4 & 4 & 4 & 3 & 11 & 10 & 91 \\
\hline Total & 145 & 122 & 127 & 111 & 141 & 129 & 413 & 362 & 88 \\
\hline
\end{tabular}

parasita da rã. O ritmo estacional é correlacionado com a temperatura da água em que o batráquio vive, sendo os niveis de parasitemias mais elevadas no verão e mais baixos no inverno. Entretanto, esses autores acreditam que a temperatura não é o único fator responsável pelos ciclos estacionais. O ritmo diurno foi influenciado pelo regime fotoperiódico ao qual as rãs eram expostas. $O$ fotoperiodismo também estava correlacionado com a variação estacional ${ }^{13} 17 \mathrm{da}$ parasitemia.

Muito importante também é o tipo de animal experimentado o que, conforme foi observado, interfere no ritmo circadiano de certas espécies de tripanossomas, como no ritmo do Trypanosoma congolonse, o qual varia também de acordo com a espécie de mamífero infectado, assim como com a cepa do tripanossoma usada, além de outros fatores 910 .
Com referencia ao $T$. cruzi, o camundongo provavelmente, não é um animal adequado para a verificação experimental da periodicidade sangüinea do parasita, principalmente por causa de sua baixa volemia que mal permite retirar quantidades de sangue suficientes para exames durante horas seguidas. $O$ camundongo também não suporta um número adequado de triatomineos nos xenos. Infelizmente, o camundongo é um dos poucos animais de laboratório onde as caracteristicas da infecção pelo $T$. cruzi já são bem conhecidas ${ }^{15}$.

Porque utilizamos camundongos brancos e cobaias, não podemos esquecer o fato de serem eles animais de laboratório e portanto artificiais, ou melhor, estranhos à biocenose do protozoário. Talvez essa seja uma das razões de termos obtido resultados diferentes daqueles observados por Deane e colabora- 


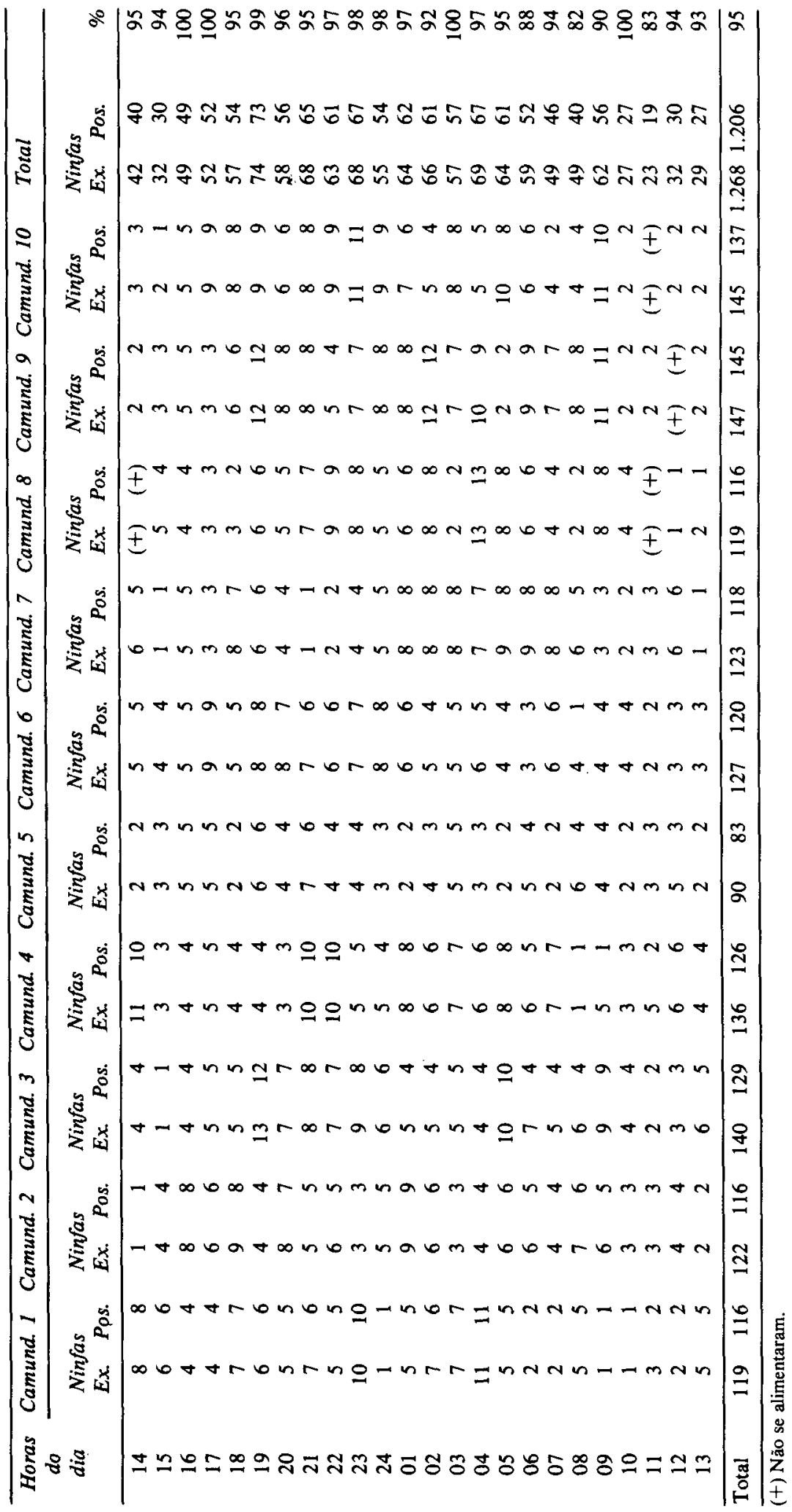


Sherlock LA. Parasitemia constante durante 24 horas consecutivas na infeç̧ão experimental pelo Trypanosoma cruzi Revista da Sociedade Brasileira de Medicina Tropical 17: 137-144, Jul-Set, 1984

Tabela 5 - Resultado de hemoscopias realizadas em 3 cobaias e 10 camundongos infectados experimentalmente pelo $\mathrm{T}$. cruzi durante 24 horas consecutivas, na fase crônica da infecção. (As cobaias e camundongos são os mesmos referidos nas Tabelas 3 e 4).

\begin{tabular}{ccc}
\hline $\begin{array}{c}\text { Hora do } \\
\text { dia }\end{array}$ & $\begin{array}{c}\text { Soma dos } \\
\text { tripanossomas } \\
\text { nas cobaias (+) }\end{array}$ & $\begin{array}{c}\text { Soma dos } \\
\text { nosipanossomas }\end{array}$ \\
\hline 14 & 3 & 0 \\
15 & 8 & 3 \\
16 & 3 & 1 \\
17 & 1 & 0 \\
18 & 3 & 0 \\
19 & 0 & 1 \\
20 & 8 & 4 \\
21 & 4 & 2 \\
22 & 6 & 1 \\
23 & 3 & 0 \\
24 & 4 & 1 \\
01 & 0 & 0 \\
02 & 4 & 0 \\
03 & 1 & 1 \\
04 & 5 & 0 \\
05 & 4 & 1 \\
06 & 3 & 0 \\
07 & 0 & 0 \\
08 & 5 & 4 \\
09 & 1 & 0 \\
10 & 0 & 0 \\
11 & 2 & 1 \\
12 & 1 & 0 \\
13 & 0 & 2 \\
\hline 12 & 5 &
\end{tabular}

$(+)$ Refere-se ao número total de tripanossomas observados por $3,09 \mathrm{~mm}^{3}$ de sangue.

dores em Callithrix 78 , para o Trypanosoma minasense, flagelado filogeneticamente próximo ao $T$. cruzi e parasita natural deste primata.

Embora não tenhamos constatado um ritmo circadiano para o $T$. cruzi, além da metodologia original que empregamos e que poderá servir de base para observações futuras a respeito, as experiências que realizamos foram proveitosas e, algumas conclusões puderam ser tiradas. Em primeiro, verificou-se que a parasitemia pelo $T$. cruzi, nos dois tipos de animais utilizados, foi constante nas 24 horas do dia, tanto na fase inicial da infecção quando o número de parasitas é elevado, como na fase crônica em que os mesmos são escassos. Como os dados mostraram, não existe um ritmo circadiano e conseqüentemente, não existe um horário melhor para a realização de hemoscopias ou xenodiganóstico em que se possa detectar melhor o parasita. Do ponto de vista prático, sabemos agora que, a demonstração do parasita pode ser feita a qualquer hora do dia, tanto através do xenodiagnóstico como da hemoscopia. Se os xenos forem repetidos num mesmo dia, as possibilidades de diagnosticar o parasita na fase crônica, são muito maiores como já haviam observado Almeida, Sherlock e Fahel ${ }^{1}$, para a infecção humana crônica.

$O$ elevado indice de positividade dos xenodiagnósticos que obtivemos nestas experiências, na fase crônica da doença, quando a hemoscopia demonstrava escassos tripanossomas circulantes, pode parecer estranho. Resultados também aparentemente inexplicáveis foram obtidos por Schenone e colaboradores $^{12}$ que, apesar da constante parasitemia do paciente que investigam, apenas $40 \%$ das ninfas que o sugaram se infectaram. $\mathrm{Na}$ realidade ${ }_{2}$ além de outros fatores inerentes à propria susceptibilidade de infecção dos triatomineos, cujos fatores são inúmeros e muitos dos quais já esclarecidos, nas nossas experiências na fase crônica, foi utilizado maior número de triatomíneos por xeno. Este fato é sabido que aumenta a positividade dos resultados 21215 , independentemente da espécie, do tamanho e do estágio evolutivo do inseto. Tal aspecto que já tem sido bastante investigado, não cabe entretanto ser discutido no presente trabalho.

\section{SUMMARY}

The hourly parasitaemia of Trypanosoma cruzi in mice with acute infection and in mice and guinea pigs with chronic infections was checked by means of hourly blood examinations and xenodiagnosis. The number of $\mathrm{T}$. cruzi in the blood and the number of positive xenodiagnosis in both acute and chronic infections was frequent and variable but not cyclical during the 24 hours of the day. T. cruzi was demonstrable at any time of the day by either blood examination or xenodiagnosis.

Key words: Trypanosoma cruzi. Hourly Parasitaemia. Circadian rhythm. Xenodiagnosis.

\section{REFERÊNCIAS BIBLIOGRÁFICAS}

1. Almeida SP, Sherlock IA, Fahel E. Novo procedimento do xenodiagnóstico na forma crônica da doença de Chagas. Memórias do Instituto Oswaldo Cruz 74: 285 $288,1976$. 
2. Almeida SP, Miles MA, Marsden PD. Verificação da susceptibilidade à infecção por Trypanosoma cruzi, dos estágios evolutivos de Rhodnius neglectus. Revista Brasileira de Biologia 33: 43-52, 1973.

3. Bardsley JE, Harmsen R. The trypanosomes of Ranidae. I. The effects of temperature and diurnal periodicity on the peripheral parasitaemia in the bulfrog (Rana catesbeiana Shaw). Canadian Journal of Zoology 47: 283-288, 1969.

4. Bardsley JE, Harmsen R. The trypanosomes of Ranidae. II. The effects of excitation and adrenalin on the peripheral parasitaemia in the bulfrog (Rana catesbeiana Shaw). Canadian Journal of Zoology 48: $1317-$ $1319,1970$.

5. Brener Z. Contribuição ao estudo da terapéutica experimental da doença de Chagas. Tese de Docência Livre, Faculdade de Odontologia e Farmácia da Universidade Federal de Minas Gerais, Belo Horizonte, 1961.

6. Cornford EM, Freeman BJ, MacInnis AJ. Physiological relationships and circadian periodicities in rodent trypanosomes. Transactions of the Royal Society of Tropical Medicine and Hygiene 70: 238-243, 1976.

7. Deane LM, Silva JE, Loures Filho L. Circardian rhythms in the parasitaemia of the primate haemoflagellate Trypanosoma minasense. Transactions of the Royal Society of Tropical Medicine and Hygiene 67: 424-425, 1973.

8. Deane LM, Silva JE da, Loures Filho L. Nycthemeral variation in parasitaemia of Trypanosoma minasense in naturally infected marmosets of the genus Callithrix (Primates, Callithricidae). Revista do Instituto de $\mathrm{Me}$ dicina Tropical de São Paulo 16: 1-6, 1974.

9. Hawking F. Circadian rhythms of Trypanosoma congolense in laboratory rodents. Transactions of the Royal Society of Tropical Medicine and Hygiene 72: 592-595, 1978.
10. Hawking F. Circadian rhythms in Trypanosoma congolense. Transactions of the Royal Society of Tropical Medicine and Hygiene 70: 170, 1976.

11. Mason G. The diurnal rhythms of Trypanosoma rotatorium in Rana clamitans: investigation of photoreceptors and physiological control. Proceeding of the Second International Congress of Parasitology, Washington D. C. USA, 1970.

12. Schenone H, Rojo M, Rojas A, Concha L. Positividad diurna y noturna del xenodiagnostico en un paciente con infección chagasica crónica de parasitemia permanente. Boletin Chileno de Parasitologia 32: 63-66, 1977.

13. Seed JR. Diurnal and seasonal rhythms in parasitaemia levels of some trypanosomes infecting Rana clamitans from Lousiania. Resumes of the Second International Congress of Parasitology 56: 311, 1970.

14. Sherlock IA. Novas observações sobre a inexistência de ritmo circadiano para Trypanosoma cruz $i$ em condiçōes experimentais. In: Resumos do XVI Congresso da Sociedade Brasileira de Medicina Tropical, Natal, RN, 1980.

15. Sherlock IA, Almeida, SP. Diferenças de susceptrbilidade à infecção com $T$. cruzi entre especcies de triatomineos alimentados em cão, tatu e camundongo infectados. Revista da Sociedade Brasileira de Medicina Tropical 7: 87-98, 1971.

16. Sherlock IA, Guitton N, Muniz, TM. Positividade durante 24 horas do xenodiagnóstico em camundongos na fase aguda da infecção pelo $T$. cruzi. In: Resumos do XIV Congresso da Sociedade Brasileira de Medicina Tropical e III Congresso da Sociedade Brasileira de Parasitologia, João Pessoa, Paraiba, 1978.

17. Shouthworth GC, Mason G, Seed JR. Studies on frog trypanosomiasis. I. A 24-Hour Cycle in the parasitaemia level of Trypanosoma rotatorium in Rana clamitans from Louisiana. The Journal of Parasitology 54: $255-258,1968$. 